\title{
RESULTADOS DA TENOTOMIA DOS MÚSCULOS ADUTORES DO QUADRIL NA PARALISIA CEREBRAL ESPÁSTICA
}

\author{
ADDUCTORS TENOTOMY IN SPASTIC CEREBRAL PALSY
}

Luiz Gabriel Betoni Guglielmetti', Ruy Mesquita Maranhão Santos', Rodrigo Góes Medea de Mendonça', Helder Henzo Yamada², Rodrigo Montezuma César de Assumpção ${ }^{3}$, Patricia Maria de Moraes Barros Fucs ${ }^{4}$

\section{RESUMO}

Objetivo: Avaliação radiográfica da evolução dos quadris submetidos à liberação de partes moles. Métodos: Avaliação retrospectiva de 101 pacientes com paralisia cerebral submetidos à liberação de partes moles, no período entre 1991 e 2006. Destes, 44 pacientes com critérios de inclusão, 23 do sexo masculino e 21 do feminino, 34 diparéticos e 10 tetraparéticos. Funcionalmente, 29 não andadores, cinco andadores domiciliares e 10 andadores comunitários. Foram mensurados o índice acetabular (IA) e índice de Reimers (IR) nas radiografias pré e pós-operatória, com tempo mínimo de seguimento de três anos. A idade média na cirurgia foi 6,4 anos. Resultados: Considerou-se bom resultado os quadris com diminuição ou aumento menor que $10 \%$ do IR (52\% deste estudo). Observou-se melhora evidente do IR, além de apresentar piores resultados nos pacientes maiores de cinco anos de seguimento pós-operatório. Conclusão: Deve-se realizar a liberação de partes moles, o mais precocemente possível, independentemente da idade, condição deambulatória, tipo clínico, IR, IA e sexo, assim que apresentar abdução menor que 30 graus clinicamente, devido aos benefícios quanto à marcha, prevenção e tratamento da subluxação, higienização e alívio da dor.

\section{ABSTRACT}

Objective: Radiographic evaluation of the evolution of hips that underwent soft-tissue release. Methods: Retrospective study of 101 spastic Cerebral Palsy patients who underwent softtissue release between 1991 and 2006. Forty-four patients met the inclusion criteria, 23 boys and 21 girls, 34 diparetic and 10 quadriparetic. Twenty-nine were non-ambulatory, 5 were ambulatory domestically and 10 ambulatory. Reimers' index (RI) and the acetabular index (AI) were measured, pre- and post-operatively, with a minimum follow-up of 3 years. The mean age at the time of surgery was 6.4 years. Results: Results were considered good if the RI was reduced or had increased less than $10 \%$. Results were good for $52 \%$ of this study. We observed a clear improvement of IR, in addition to presenting the worst results, in patients older than five years in follow-up. Conclusion: Soft-tissues releases should be done as soon as the patient clinically presents less than $30^{\circ}$ of hip abduction, regardless the age, level of functioning, clinical type, $R I, A I$ and sex, to help patients in the prevention and treatment of the subluxation, hygiene, and pain relief.

Keywords - Cerebral palsy; Hip dislocation

Descritores - Paralisia cerebral; Luxação do quadril

1 - Médico Residentes do Departamento de Ortopedia e Traumatologia da Santa Casa de Misericórdia de São Paulo, Faculdade de Ciências Médicas da Santa Casa de São Paulo - Pavilhão "Fernandinho Simonsen".

2 - Médico Assistente do Departamento de Ortopedia e Traumatologia da Santa Casa de Misericórdia de São Paulo, Faculdade de Ciências Médicas da Santa Casa de São Paulo - Pavilhão "Fernandinho Simonsen".

3 - Médica Assistente e Chefe do Grupo das Doenças Neuromusculares do Departamento de Ortopedia e Traumatologia da Santa Casa de Misericórdia de São Paulo, Faculdade de Ciências Médicas da Santa Casa de São Paulo - Pavilhão "Fernandinho Simonsen".

4 - Médica Assistente e Chefe de Clínica Adjunto- Doutora Departamento de Ortopedia e Traumatologia da Santa Casa de Misericórdia de São Paulo, Faculdade de Ciências Médicas da Santa Casa de São Paulo - Pavilhão "Fernandinho Simonsen".

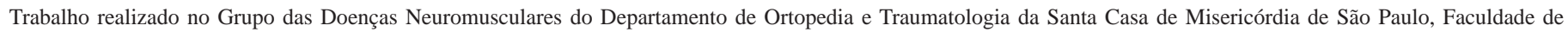
Ciências Médicas da Santa Casa de São Paulo - Pavilhão “Fernandinho Simonsen”. Diretor: Prof. Dr. Osmar Avanzi.

Correspondência: Patricia Maria de Moraes Barros Fucs, Rua Dr. Cesário Motta Jr., 112 - 01227-900 - São Paulo, SP. E-mail: dot.neuromuscular@santacasasp.org.br 


\section{INTRODUÇÃO}

Crianças com Paralisia Cerebral podem apresentar subluxação ou luxação do quadril devido a espasticidade ${ }^{(1,2)}$. Acredita-se que seja resultado do desequilíbrio muscular, causador de posições anômalas do quadril em adução, flexão e rotação interna ${ }^{(3)}$. A gravidade dos deslocamentos do quadril é relacionada ao grau de espasticidade e de funcionalidade, sendo a prevalência de $80 \%$ (35 de 44 crianças) desta condição ser mais frequente em crianças não deambuladoras e com padrão tetraparético ${ }^{(4)}$. Os efeitos secundários da subluxação e deslocamento espásticos incluem problemas no posicionamento, higiene pessoal, marcha e dor. Além disso, a posição em adução do quadril contribui para o desenvolvimento de obliquidade pélvica e subsequente escoliose ${ }^{(5,6)}$.

Diferentes procedimentos cirúrgicos, incluindo cirurgias de partes moles e ósseas podem ser usadas para o tratamento dessas condições, dependendo da idade, gravidade e da alteração do quadril. Cirurgias de partes moles (ressecções musculares e tenotomias) agem na prevenção da subluxação e luxação do quadril espástico ${ }^{(7,8)}$. Entretanto, existem dúvidas quanto à idade, as indicações dos procedimentos cirúrgicos e os músculos envolvidos, assim como a condução pós-operatória ${ }^{(9)}$, também, quanto a durabilidade do resultado obtido.

O objetivo deste estudo foi avaliar de modo radiográfico a evolução do quadril de crianças com Paralisia Cerebral diparética e tetraparética, após realização de liberação de partes moles (tenotomia dos músculos adutores e grácil).

\section{CASUÍSTICA E MÉTODOS}

A metodologia consistiu na avaliação retrospectiva de 101 pacientes com diagnóstico de Paralisia Cerebral diparética e tetraparética, submetidos à cirurgia de liberação de partes moles (tenotomia dos músculos adutores e grácil), no período compreendido entre 1991 e 2006, pelo Grupo de Doenças Neuromusculares do Departamento de Ortopedia e Traumatologia da Faculdade de Ciências Médicas da Santa Casa de São Paulo -"Pavilhão Fernandinho Simonsen”. Destes, 44 pacientes que respeitavam todos os critérios de inclusão (seguimento clínico e radiográfico completos), sendo 23 do sexo masculino (M) e 21 do sexo feminino (F), 34 diparéticos (Dipa) e 10 tetraparéticos (Tetra). Foi avaliada a situação da marcha pré-operatória, que consistiu em 29 não andadores (NA), cinco andadores domiciliares (AD) e
10 andadores comunitários (AC).

A idade média dos pacientes na ocasião da realização da cirurgia foi de 6,4 anos, variando entre um e 20 anos de idade.

Indicou-se a cirurgia para os pacientes com abdução menor que 30 graus, pesquisada com os quadris em extensão e ao reflexo do estiramento.

A análise radiográfica foi realizada utilizando-se como parâmetros o índice de Reimers (IR) ${ }^{(7)}$ e o índice acetabular (IA) ${ }^{(10)}$. O índice de Reimers ${ }^{(7)}$ consiste na percentagem de migração da cabeça femoral, obtido através da percentagem de cabeça femoral lateral à linha de Perkins em relação ao diâmetro total da epífise femoral proximal. Reimers considerou que até três anos de idade, o quadril normal apresenta-se com a cabeça femoral completamente coberta pelo acetábulo. Dos quatro aos 16 anos, considerou normal até 5\% lateral à linha de Perkins. Em pacientes com Paralisia Cerebral, considera-se subluxado o quadril com mais de 33\% de migração, e luxado com $100 \%{ }^{(7)}$. Dividimos grupos para estratificar a condição radiográfica pré-operatória, sendo: normal IR $<20 \%$, quadril em risco IR 20 - 32\%, subluxação IR 33 - 89\% e, luxação IR > 90\%, conforme descrito por Terjesen et al ${ }^{(11)}$.

Os parâmetros radiográficos foram medidos nas radiografias pré-operatória e na mais recente obtida, na incidência anteroposterior da bacia. O tempo mínimo entre a radiografia pré-operatória e a pós-operatória foi de três anos, máximo de 15 anos, com uma média de cinco anos e 11,5 meses (Figuras 1, 2, 3 e 4).

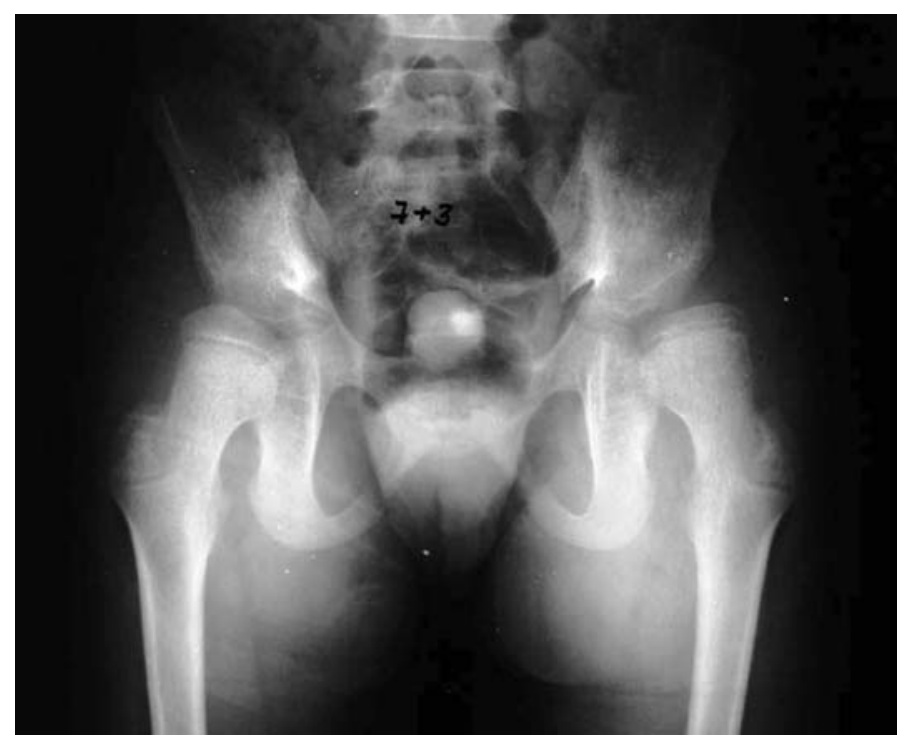

Figura 1 - Paciente do sexo masculino (caso $n^{\circ} 1$ ), idade sete anos e três meses, radiografia anteroposterior da bacia préoperatória 


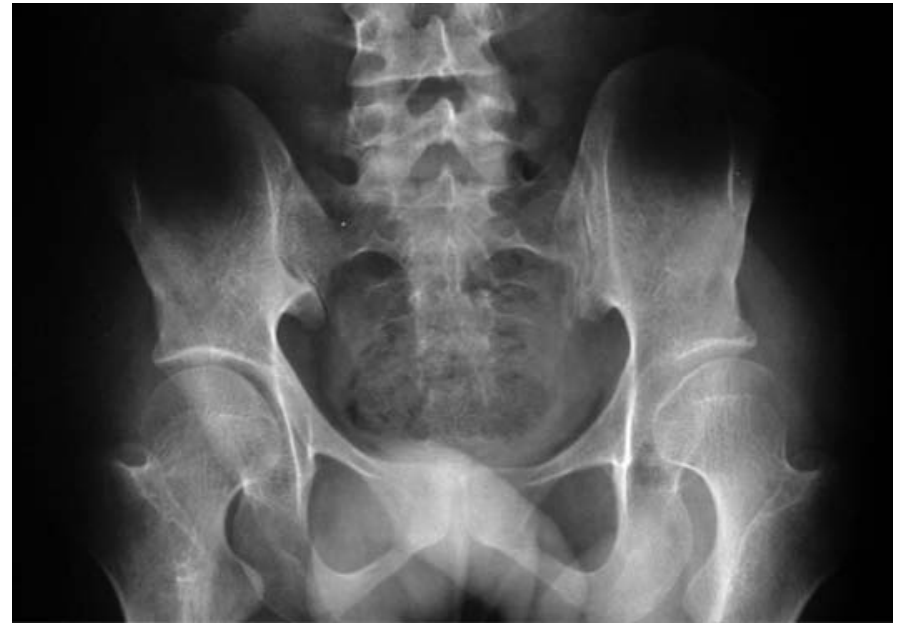

Figura 2 - Paciente do sexo masculino (caso $n^{\circ}$ 1), idade 21 anos e dois meses, radiografia anteroposterior da bacia pósoperatória seis anos e 11 meses

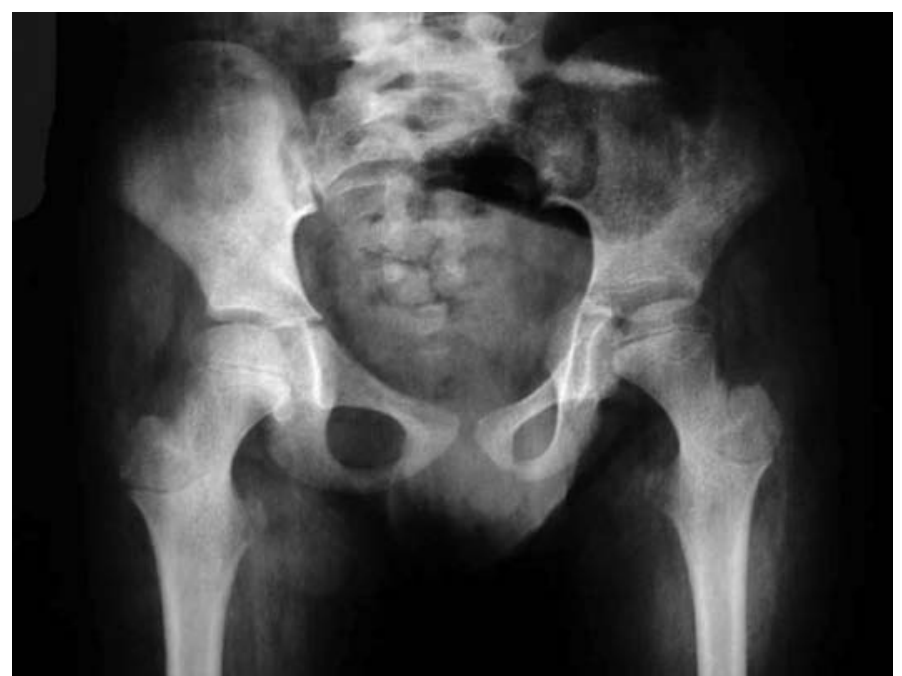

Figura 3 - Paciente do sexo masculino (caso $n^{\circ} 2$ ), idade oito anos e dois meses, radiografia anteroposterior da bacia préoperatória

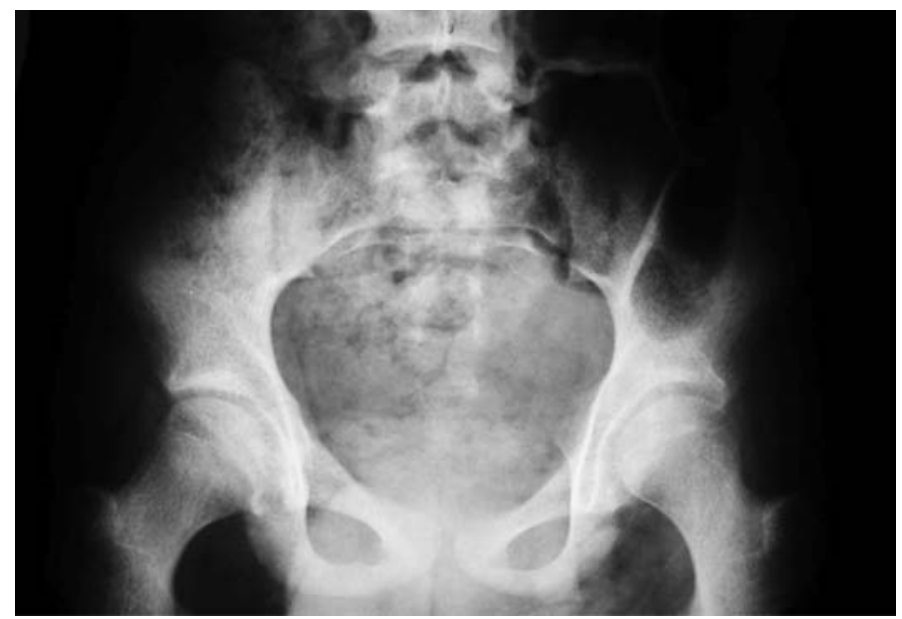

Figura 4 - Paciente sexo masculino (caso $n^{\circ} 2$ ), idade 11 anos e oito meses, radiografia anteroposterior da bacia pós-operatória três anos e seis meses
Realizou-se a tenotomia dos músculos adutores bilateralmente (adutor longo, curto e grácil) e os pacientes foram mantidos em gesso cruropodálico bilateral com barra de abdução por três semanas, seguido de tratamento fisioterápico.

Os critérios de avaliação dos resultados, segundo Kalen e Bleck ${ }^{(12)}$ foram considerados bons resultados quando o IR diminuía ou aumentava até $10 \%$; se aumentado mais de $10 \%$, como mau resultado. Analisamos os quadris isoladamente e os pacientes como um todo, neste caso, considerando o lado mais afetado.

Para a avaliação das variáveis qualitativas entre si foram utilizados os testes do Qui-quadrado ou exato de Fisher. Já a análise das variáveis pré e pós-operatórias, para avaliação da evolução, foi utilizado o teste de McNemar. Os softwares utilizados foram o Epi-info 6.0 e o SPSS para Windows (v. 13), e considerou-se significância estatística quando $\mathrm{p}<0,05$.

\section{RESULTADOS}

O tempo de seguimento médio foi de 5,9 anos.

Do grupo de 44 pacientes (88 quadris), bom resultado foi obtido em 23 pacientes (52\%) e mau resultado em 21 (48\%). Quanto à função deambulatória, dos 10 pacientes AC, sete obtiveram bom resultado (70\%); dos cinco AD, dois obtiveram bom resultado (40\%), e dos 29 NA, 14 apresentaram bons resultados (48\%), $\mathrm{P}=0,417$ (teste do Qui-quadrado) (Tabela 1).

Tabela 1 - Resultados de acordo com a função motora

\begin{tabular}{c|c|c|c}
\hline \multirow{2}{*}{ Função } & \multicolumn{3}{|c}{ Resultado } \\
\cline { 2 - 4 } & Bom & Mau & Total \\
\hline AC & 7 & 3 & 10 \\
\hline AD & 2 & 3 & 5 \\
\hline NA & 14 & 15 & 29 \\
\hline Total & 23 & 21 & 44 \\
\hline Fonte I: SAME-ISCMSP \\
AC: andador comunitário; AD: andador domiciliar; NA: não-andador
\end{tabular}

Quanto aos tipos de Paralisia Cerebral espástica, 34 pacientes eram diparéticos, obtendo bom resultado em 18 (53\%). Já no grupo dos tetraparéticos, cinco (50\%) apresentaram bom resultado. Foi avaliada, também, a idade do paciente na época da cirurgia; dividiu-se em dois grupos, os de quatro anos ou menos e o outro, formado por pacientes com mais de quatro anos. Bom resultado foi obtido em sete (50\%) dos 14 , do primeiro grupo citado acima, e 16 (53\%) dos 30, do outro grupo, não demonstrando tendências prognósticas quanto a idade da cirurgia, $\mathrm{p}=0,837$ (Qui-quadrado), conforme representado na Figura 5. 


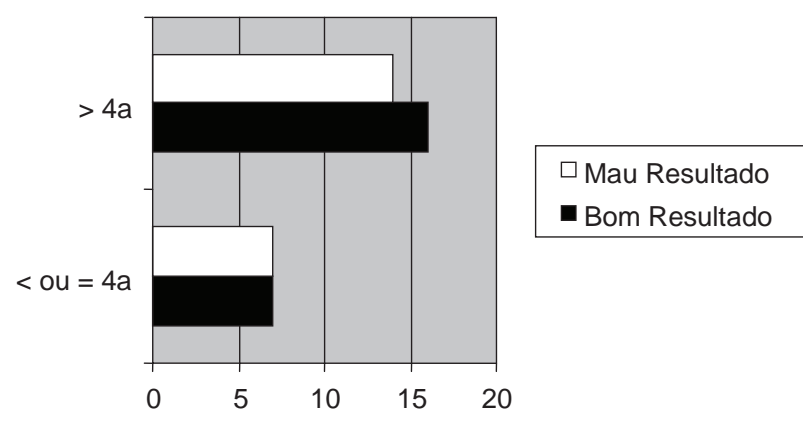

Figura 5 - Resultados segundo a idade do paciente por ocasião

Foram comparados os resultados dos pacientes com seguimento menor e maior ou igual a cinco anos. Observou-se que no grupo menor que cinco anos, 13 (65\%) pacientes obtiveram bom resultado, enquanto no grupo maior que cinco anos, 10 (42\%) apresentaram bom resultado, evidenciando piora radiográfica em longo prazo. A análise estatística quanto ao sexo ( $\mathrm{M}$ e F), tipo de Paralisia Cerebral (Dipa e Tetra) (Figura 6), função deambulatória (AC, AD e NA), idade na cirurgia (menor ou igual a quatro anos e maior que quatro anos) e tempo de seguimento (menor que cinco anos e maior ou igual a cinco anos ) (Figura 7), comparados aos resultados (bom e ruim), não foi significativa $(p>0,05)$.

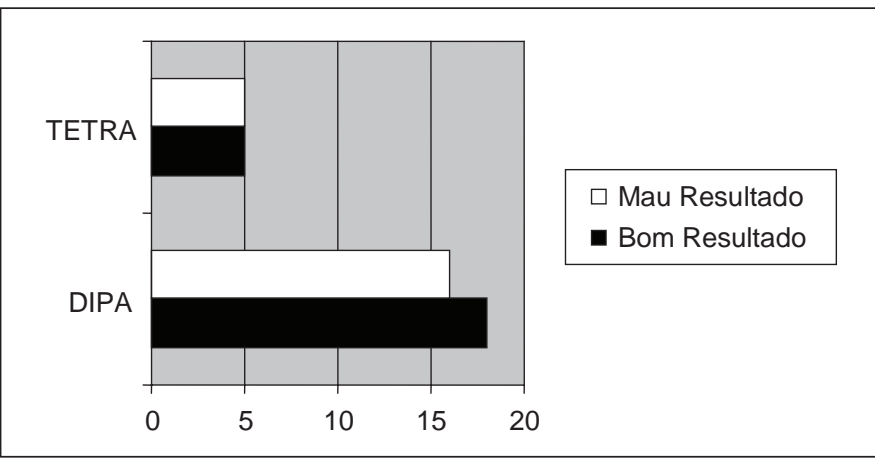

Figura 6 - Resultados da cirurgia comparado ao tipo funcional da paralisia cerebral

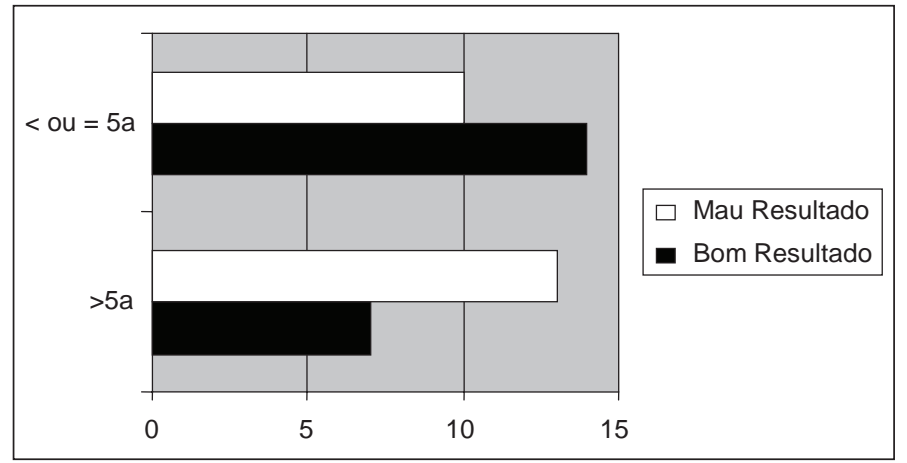

Figura 7 - Resultado da cirurgia de acordo com o tempo de seguimento
Os quadris foram avaliados individualmente e por paciente, considerando o pior lado. Em relação ao lado direito, 21 apresentavam inicialmente quadril normal, 10 em risco e 13 subluxados; dos normais, 10 (48\%) obtiveram bom resultado; dos em risco, oito (80\%) e dos subluxados, 12 (92\%), apresentando significância estatística, $\mathrm{p}=0,016$ (Qui-quadrado). Não foi verificada tal significância no lado esquerdo, $\mathrm{p}=0,853$ (Quiquadrado), porém evidenciou-se bom resultado em 11 (73\%) dos quadris normais, 12 (80\%) dos em risco e 10 (71\%) dos subluxados (Tabelas 2 e 3). Avaliando o paciente, ao considerar o pior lado como resultado final (Tabela 4) obteve-se bom resultado em cinco (42\%) dos quadris normais, oito (57\%) dos em risco e em nove (50\%) dos subluxados, $\mathrm{p}=$ 0,687 (Qui-quadrado).

Tabela 2 - Resultados de acordo com o índice de Reimers do lado direito pré-operatório

\begin{tabular}{l|c|c|c}
\hline \multicolumn{4}{c}{ Resultado } \\
\hline IR D pré & Bom & Mau & Total \\
\hline Normal & 10 & 11 & 21 \\
\hline Risco & 8 & 2 & 10 \\
\hline Subluxado & 12 & 1 & 13 \\
\hline Total & 30 & 14 & 44 \\
\hline $\begin{array}{l}\text { Fonte I: SAME-ISCMSP } \\
\text { IR: índice de Reimers; D: lado direito }\end{array}$
\end{tabular}

Tabela 3 - Resultados de acordo com o índice de Reimers do lado esquerdo pré-operatório

\begin{tabular}{c|c|c|c}
\hline \multicolumn{3}{c}{ Resultado } \\
\hline IR E pré & Bom & Mau & Total \\
\hline Normal & 11 & 4 & 15 \\
\hline Risco & 12 & 4 & 15 \\
\hline Subluxado & 10 & 4 & 14 \\
\hline Total & 33 & 11 & 44 \\
\hline Fonte I: SAME-ISCMSP \\
IR: índice de Reimers; E: lado esquerdo
\end{tabular}

Tabela 4 - Resultados de acordo com o índice de Reimers total pré-operatório

\begin{tabular}{c|c|c|c}
\hline \multicolumn{3}{c}{ Resultado } \\
\hline IR pré-total & Bom & Mau & Total \\
\hline Normal & 5 & 7 & 12 \\
\hline Risco & 8 & 6 & 14 \\
\hline Subluxado & 9 & 9 & 18 \\
\hline Total & 22 & 22 & 44 \\
\hline Fonte I: SAME-ISCMSP & & &
\end{tabular}

IR: índice de Reimers 
Quando comparados quanto à apresentação (normal, risco e subluxado) inicial e final, obteve-se $\mathrm{p}>0,05$ (McNemar) em ambos os lados, não apresentando significância estatística (Tabelas 5, 6 e 7).

Tabela 5 - Resultados de acordo com o índice de Reimers do lado direito (D) pré e pós-operatórios

\begin{tabular}{l|c|c|c|c}
\hline \multicolumn{5}{c}{ IR pós-operatório } \\
\hline IR D pré-operatório & Normal & Risco & Subluxado & Total \\
\hline Normal & 14 & 6 & 1 & 21 \\
\hline Risco & 4 & 6 & 0 & 10 \\
\hline Subluxado & 3 & 4 & 6 & 13 \\
\hline Total & 21 & 16 & 7 & 44 \\
\hline
\end{tabular}

Fonte I: SAME-ISCMSP

IR: índice de Reimers; D: lado direito

Tabela 6 - Resultados de acordo com o índice de Reimers do lado esquerdo $(\mathrm{E})$ pré e pós-operatórios

\begin{tabular}{l|c|c|c|c}
\hline \multicolumn{5}{c}{ IR pós-operatório } \\
\hline IR E pré-operatório & Normal & Risco & Subluxado & Total \\
\hline Normal & 15 & 0 & 0 & 15 \\
\hline Risco & 4 & 8 & 3 & 15 \\
\hline Subluxado & 2 & 7 & 5 & 14 \\
\hline Total & 21 & 15 & 8 & 44 \\
\hline Fontel:SAME-ISCMSP & & & &
\end{tabular}

IR: índice de Reimers; E: lado esquerdo

Tabela 7 - Resultados de acordo com o IR Total pré e pósoperatório

\begin{tabular}{l|c|c|c|c}
\hline \multicolumn{5}{c}{ Resultado IR total pós-operatório } \\
\hline IR Total pré-operatório & Normal & Risco & Subluxado & Total \\
\hline Normal & 11 & 1 & 0 & 12 \\
\hline Risco & 3 & 8 & 3 & 14 \\
\hline Subluxado & 3 & 6 & 9 & 18 \\
\hline Total & 17 & 15 & 12 & 44 \\
\hline Fonte I: SAME-ISCMSP & & & &
\end{tabular}

IR: índice de Reimers

\section{DISCUSSÃO}

Os 44 pacientes selecionados, retrospectivamente, foram avaliados para demonstrar evidências de quando se deve realizar tenotomia dos músculos adutores, se há indícios prognósticos e para comparar com resultados da literatura. Deve-se considerar que a tenotomia dos músculos adutores não tem como objetivo somente a prevenção, mas, também, o tratamento do quadril subluxado.
Existem outros benefícios como melhora da marcha e do ortostatismo, aumento da abdução, melhora da simetria do quadril, facilitação da higiene e alívio da dor ${ }^{(11)}$.

Existem algumas formas de definir o resultado. Segundo Kalen e Bleck ${ }^{(12)}$, bom resultado ocorre quando há diminuição ou aumento menor que $10 \%$ do IR. Silver et $a^{(13)}$ consideraram insatisfatório, os pacientes que necessitaram nova cirurgia para adquirir estabilidade do quadril (partes moles ou óssea). Turkey e Lee ${ }^{(9)}$ adicionaram a esta, que IR maior que $80 \%$ como resultado insatisfatório. Miller et al ${ }^{(14)}$ classificaram como satisfatório quando IR menor ou igual a 39\% e insatisfatório quando maior que $40 \%$. As três classificações citadas acima merecem críticas. Segundo a classificação de Miller et $a l^{(14)}$, um quadril com diminuição evidente do IR, porém se mantendo maior que $40 \%$ é considerado como resultado insatisfatório. De acordo com Silver et $a l^{(13)}$, um paciente que realiza tenotomia dos músculos adutores, já com quadril gravemente displásico que, provavelmente, sofrerá cirurgia de partes ósseas em breve, é considerado como insatisfatório. Segundo Kalen e Bleck ${ }^{(12)}$, um paciente com quadril normal, que apresenta aumento do IR maior que $10 \%$, porém se mantendo com quadril normal, é considerado resultado ruim. Baseado nesta análise, utilizou-se no presente estudo a classificação de Kalen e Bleck ${ }^{(12)}$, porém, também foi avaliada a situação inicial em relação à final (Tabelas 5, 6 e 7). Ao avaliar o lado direito, observou-se bom resultado em $92 \%$ dos subluxados, $80 \%$ dos em risco e em apenas $48 \%$ dos normais. Porém, ao avaliar a situação do quadril inicial com a final, observamos que dos 21 quadris normais, 14 mantiveram-se normais, seis foram para o grupo em risco e apenas um subluxou (Tabela 5), demonstrando a importância de avaliarmos os resultados através de diferentes métodos. Estes bons resultados se repetem ao avaliar o lado esquerdo (Tabela 6). Comparando tais resultados com a literatura, há concordância com a conclusão de Presedo et $a^{(15)}$ que a liberação de partes moles, efetivamente, pode tratar e prevenir a subluxação dos quadris com Paralisia Cerebral. Obviamente, avaliando o paciente considerando o pior quadril, temos resultados inferiores, como observado em outras publicações $^{(11)}$ (Tabelas 4 e 7). Vale mencionar que é impossível estabelecer um grupo controle neste estudo, pois existem outras indicações para esta cirurgia além da prevenção da subluxação.

Mediu-se no presente estudo o índice acetabular, encontrando uma média de 23,82 graus no período pré-operatório. 
Não foi encontrada diferença estatística ao comparar os resultados dos pacientes com IA $\leq 27,5$ com os $\mathrm{IA}>27,5$, apresentando uma discreta tendência a melhores resultados nos quadris com IA menor, tal como demonstrado por Terjesen et $a l^{(11)}$.

Deve-se considerar que ambas mensurações radiográficas são sujeitas a variações quando medida por diferentes observadores ou mesmo pelo próprio mensurador. Parrott et al ${ }^{(16)}$ concluíram que se deve aceitar aproximadamente 8\% de desvio padrão para o IR e quatro graus para o IA.

Em relação à idade dos pacientes na época da cirurgia, Kalen e Bleck ${ }^{(12)}$ encontraram melhores resultados em pacientes mais jovens, e recomendaram que seja realizada antes dos cinco anos. Sharrard et $a^{(17)}$ e Onimus et $a l^{(18)}$, também encontraram tais resultados. Por outro lado, o presente estudo não encontrou diferença entre os pacientes operados antes e após os quatro anos de idade, de acordo com outros trabalhos (Silver et al ${ }^{(13)}$, Cottalorda et $a l^{(19)}$, Turker e Lee ${ }^{(9)}$, Terjesen ${ }^{(11)}$ e Presedo et $\left.a l^{(15)}\right)$.

Não foi evidenciada significância estatística em relação ao estado deambulatório inicial, tal como os resultados de Silver et $a l^{(13)}$ e Cottalorda et $a l^{(19)}$. No presente estudo, porém, foi observado bom resultado em 70\% dos deambuladores comunitários contra 40\% nos deambuladores domiciliares e $48 \%$ dos não deambuladores, demonstrando uma tendência ao melhor resultado nos deambuladores comunitários.
O tempo de seguimento é variável importante para análise dos resultados, sendo frequentemente discutido em publicações anteriores. Reimers et $a l^{(7)}$ e Onimus et $a l^{(18)}$ apresentaram um seguimento médio de três anos. Kalen e Bleck ${ }^{(12)}$, Silver et $a^{(13)}$, Miller et al ${ }^{(14)}$, quatro a cinco anos. Turker e Lee ${ }^{(9)}$ demonstraram que bons resultados deterioravam com um seguimento mais longo. Terjesen et al ${ }^{(11)}$ recomendaram um seguimento mínimo de seis anos para avaliação dos resultados. Espera-se tal piora devido ao fato de que a evolução para subluxação não estabiliza até a maturidade esquelética ${ }^{(9)}$. Baseado nestes dados, dividimos nosso pacientes em dois grupos, o primeiro com seguimento maior ou igual a cinco anos e um segundo com seguimento menor que cinco anos. Foi observada, realmente, uma tendência a piores resultados para os pacientes com maior tempo de seguimento, porém, sem significância estatística (Figura 7).

\section{CONCLUSÃO}

Os resultados radiográficos obtidos no presente estudo permitem concluir que se deve realizar a cirurgia de partes moles o mais precocemente possível, independentemente da idade, condição deambulatória, tipo de espasticidade, IA, IR e sexo, nos pacientes com Paralisia Cerebral que clinicamente apresentem abdução do quadril menor de 30 graus devido aos benefícios quanto à marcha, higiene, alívio da dor, prevenção e tratamento da subluxação.

\section{REFERÊNCIAS}

1. Bleck EE. The hip in cerebral palsy. Orthop Clin North Am. 1980;11(1):79-104.

2. Hoffer MM, Stein GA, Koffman M, Prietto M. Femoral varus-derotation osteotomy in spastic cerebral palsy. J Bone Joint Surg Am. 1985;67(8):1229-35.

3. Miller F, Slomczykowski M, Cope R, Lipton GE. Computer modeling of the pathomechanics of spastic hip dislocation in children. J Pediatr Orthop. 1999;19(4):486-92.

4. Howard CB, McKibbin B, Williams LA, Mackie I. Factors affecting the incidence of hip dislocation in cerebral palsy. J Bone Joint Surg Br. 1985;67(4):530-2.

5. Bagg MR, Farber J, Miller F. Long-term follow-up of hip subluxation in cerebral palsy patients. J Pediatr Orthop. 1993;13(1):32-6.

6. Abel MF, Blanco JS, Pavlovich L, Damiano DL. Asymmetric hip deformity and subluxation in cerebral palsy: an analysis of surgical treatment. J Pediatr Orthop. 1999;19(4):479-85.

7. Reimers J. The stability of the hip in children. A radiological study of the results of muscle surgery in cerebral palsy. Acta Orthop Scand Suppl. 1980;184:1-100.

8. Turker RJ, Lee R. Adductor tenotomies in children with quadriplegic cerebral palsy: longer term follow-up. J Pediatr Orthop. 2000;20(3):370-4.

9. Miller F, Bagg MR. Age and migration percentage as risk factors for progression in spastic hip disease. Dev Med Child Neurol. 1995;37(5):449-55.

10. Tönnis D. Normal values of the hip joint for the evaluation of $X$-rays in children and adults. Clin Orthop Relat Res. 1976;(119):39-47.

11. Terjesen T, Lie GD, Hyldmo AA, Knaus A. Adductor tenotomy in spastic cerebral palsy. A long-term follow-up study of 78 patients. Acta Orthop. 2005;76(1):128-37.

12. Kalen V, Bleck EE. Prevention of spastic paralytic dislocation of the hip. Dev Med Child Neurol. 1985 Feb;27(1):17-24.

13. Silver RL, Rang M, Chan J, de la Garza J. Adductor release in nonambulant children with cerebral palsy. J Pediatr Orthop. 1985;5(6):672-7.

14. Miller F, Cardoso Dias R, Dabney KW, Lipton GE, Triana M. Soft-tissue release for spastic hip subluxation in cerebral palsy. J Pediatr Orthop. 1997;17(5):571-84.

15. Presedo A, Oh CW, Dabney KW, Miller F. Soft-tissue releases to treat spastic hip subluxation in children with cerebral palsy. J Bone Joint Surg Am. 2005;87(4):832-41.

16. Parrott J, Boyd RN, Dobson F, Lancaster A, Love S, Oates J, et al. Hip displacement in spastic cerebral palsy: repeatability of radiologic measurement. J Pediatr Orthop. 2002;22(5):660-7.

17. Cottalorda J, Gautheron V, Metton G, Charmet E, Maatougui K, Chavrier Y. Predicting the outcome of adductor tenotomy. Int Orthop. 1998;22(6):374-9.

18. Sharrard WJ, Allen JM, Heaney SH. Surgical prophylaxis of subluxation and dislocation of the hip in cerebral palsy. J Bone Joint Surg Br. 1975;57(2):160-6.

19. Onimus M, Allamel G, Manzone P, Laurain JM. Prevention of hip dislocation in cerebral palsy by early psoas and adductors tenotomies. J Pediatr Orthop. 1991;11(4):432-5. 